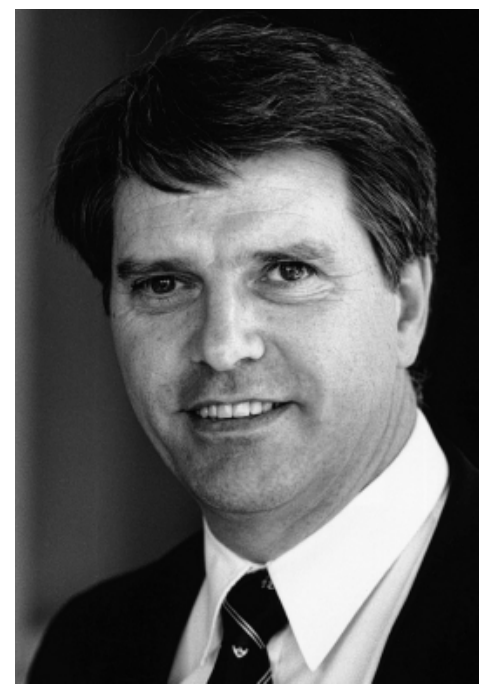

\title{
Oui aux bilatérales!
}

\author{
Y. Guisan
}

Correspondance:

Dr Yves Guisan, vice-président FMH

Conseiller national radical

Hôpital du Pays d'Enhaut

CH-1837 Château-d'Oex

Une partie des signatures à l'origine du référendum contre les accords bilatéraux provient des milieux médicaux. Certains médecins semblent craindre en effet que leurs homologues européens n'affluent en masse dans notre pays pour leur faire concurrence. Ces craintes sont injustifiées et l'expérience accumulée depuis près de 10 ans dans l'UE le confirme. Pour s'assurer toutefois qu'une telle éventualité ne se produise pas, les Chambres fédérales ont pris des mesures restrictives.

Le Conseil fédéral sera en mesure de limiter le nombre de médecins admis à pratiquer à charge de l'assurance-maladie obligatoire pour autant qu'il soit démontré qu'une l'augmentation des coûts de santé observée soit principalement la conséquence de la pléthore médicale. Cette mesure touchera aussi bien les suisses que nos collègues de la Communauté, toute disposition discriminatoire étant fondamentalement contraire aux principes européens. Mais elle sera limitée à une période de trois ans. Si l'autorisation de pratiquer de principe ne saurait être refusée dès le moment où les conditions sont remplies (équivalence des diplômes), rien n'empêche néanmoins le Conseil fédéral et les cantons de prendre des mesures restrictives en ce qui concerne la pratique à charge de l'assurance sociale. C'est d'ailleurs l'usage dans l'UE. Il est possible de s'installer partout, mais l'admission à la sécurité sociale se heurte à des obstacles administratifs divers, dont le principal est simplement le nombre de postes disponibles et pour lesquels il y a toujours des dizaines de candidatures étant donné le chômage ambiant.

\section{Les crédits bancaires sont déjà loin d'être évidents}

Mais indépendamment de ces dispositions légales et de la libre circulation des personnes, les obstacles à l'installation sont nombreux, même pour les jeunes consœurs et confrères actuellement en formation dans nos hôpitaux. Ils sont actuellement plus de 2000 dans notre pays. A moins d'avoir acquis une certaine notoriété à la suite de plusieurs années de pratique dans un poste hospitalier à haute responsabilité, ils vont se heurter aux mêmes difficultés de financement que les collègues en provenance directement de l'étranger. Il devra payer les prix suisses pour ses locaux et leurs équipements, et des salaires suisses à son personnel. En outre il faudra acquérir une clientèle, ce qui est tout sauf évident en l'absence de relations établies de longue date sur place. Enfin le marché est largement saturé dans les grandes villes, dans les bourgades ou villages d'une certaine importance, ainsi que dans la plupart des spécialités. Dans ces circonstances il est loin d'être certain d'obtenir facilement un crédit bancaire appelé à couvrir non seulement les investissements d'installation, mais encore les charges d'exploitation pendant une durée qui peut facilement s'étendre sur deux à trois ans ou même davantage. Déjà maintenant, les banquiers ne se pressent pas au portillon et exigent de solides garanties pour financer ce genre d'opération dont les risques sont loin d'être négligeables.

\section{La reconnaissance mutuelle des diplômes facilite le retour dans son pays}

Il ne faut donc probablement pas s'attendre à ce que la majorité des médecins assistants et chefs de cliniques européens s'établissent en Suisse. Les bilatérales ont le mérite essentiel que la formation reçue dans nos établissements sera désormais également reconnue par l'UE, ce qui facilitera leur retour éventuel dans leur pays d'origine. Ce n'était pas nécessairement le cas jusqu'à présent et souvent il ne leur restait guère d'autre issue que d'acquérir la nationalité suisse ou d'obtenir «à l'usure» l'autorisation de pratiquer en faisant valoir leur formation, ce qui bien entendu ne sera plus possible à l'eau à l'avenir. L'ensemble de ces circonstances sur le plan légal et administratif devrait être de nature à rassurer nos collègues frontaliers en particulier. 


\section{La pléthore est un problème indépendant des bilatérales}

Le problème de la pléthore est une question plus générale qui préoccupe vivement le comité central. Jusqu'à présent il n'a pas été possible de rencontrer une volonté politique suffisante pour s'attaquer au fonds du problème. Les conséquences éventuellement possibles des bilatérales ont eu indiscutablement un effet de sensibilisation. Si une réglementation pour l'admission en faculté ne dépasse pour le moment pas le stade du test d'aptitude, par contre la formation postgraduée est en voie de subir des modifications plus importantes. Les exigences de qualité et d'accréditation vont amener sans doute une diminution du nombre de postes. La planification hospitalière va être la source de réductions supplémentaires. Un groupe de travail comportant la FMH, H+ et la CDS sont en train de se pencher sur ces questions. L'évolution se fait certainement en direction d'un plus grand nombre de médecins hospitaliers plein temps et d'une diminution du nombre de places de formation. Cette situation engendre certainement une inquiétude légitime de nos jeunes consœurs et confrères. Mais pas plus que leurs aînés ils n'ont intérêt à des déséquilibres majeurs entre le nombre de places de formation et les possibilités de débouchés par la suite.

\section{La relation médecin-malade a des attaches culturelles fortes}

On compte à peu près un million de médecins en Europe. L'expérience de la libre circulation dans l'UE a montré que malgré un chômage endémique important de plusieurs milliers de médecins dans la plupart des pays membres aucun mouvement d'importance ne s'est produit à la recherche de conditions plus favorables, même dans les quelques niches disponibles. La relation médecin-malade a des attaches culturelles particulièrement fortes. Seuls une soixantaine de milliers de médecins ont décidé de quitter leur pays d'origine, dans la règle pour s'établir sur les rives de la Méditerranée à l'occasion de leur retraite, et sans émarger à l'assurance sociale. Ils se contentent en général d'une pratique privée limitée à quelques services rendus à leurs compatriotes sur place.

\section{L'attractivité de la Suisse en question - attention au nombrilisme!}

La majorité des signatures médicales au référendum proviennent de régions ou règne déjà un fort courant eurosceptique. Plus que de craintes pour eux-mêmes, a fortiori dans un milieu fortement traditionaliste, il est probable qu'il s'agit plutôt d'un effet de solidarité des médecins avec les préoccupations de leurs patients et à la conviction fortement ancrée que la Suisse est par définition attractive. Ce n'est certainement plus le cas comme on se l'imagine. Les avantages fiscaux sont de plus en plus relatifs. Les obstacles administratifs à la construction et au développement industriel et commercial sont loin d'être négligeables. Le maintien de la prospérité repose davantage sur le maintien de l'acquis avec l'exploitation avec succès de quelques créneaux particuliers que sur la création de nouvelles entreprises. L'introduction de l'Euro par exemple n'a pas débouché sur un déferlement sur notre monnaie refuge comme nombre de Cassandre, et pas les moindres, le prédisaient. Les temps ont certainement changé. Le repli sur soi n'a jamais été la source d'aucun développement économique. Nous ne bénéficierons de la relance actuellement en cours que dans une mesure limitée à défaut d'un esprit d'ouverture dont les bilatérales sont un élément incontournable

\section{Les médecins suisses ont aussi tout intérêt aux bilatérales}

Les échanges et les contacts bénéficient à tous. De tout temps nos étudiants ont eu besoin de sortir de nos murs et de découvrir de nouveaux horizons, mais cela ne pouvait que difficilement dépasser des stages limités. Même les titres post-gradués obtenus dans de prestigieuses universités étrangères n'étaient pas nécessairement pris en considération. Nos futurs jeunes consœurs et confrères vont pouvoir se former dans les universités et les hôpitaux de l'UE sans rencontrer les difficultés de reconnaissance mutuelle des diplômes comme c'est le cas actuellement. La libre circulation ouvre aussi la possibilité pour les Suisses de poursuivre une multitude de carrières dans les pays de l'UE, pourquoi pas cliniques ou de praticien au gré des choix personnels, mais surtout scientifiques ou académiques, ce qui jusqu'à présent n'entrait en ligne de compte que pour les ténors d'une spécialité internationalement reconnus. De plus les médecins et les professionnels de la santé bénéficient aussi de la prospérité générale. Ils ont intérêt autant que le reste de la population à la normalisation de nos relations économiques avec l'UE et à l'ouverture de nouvelles perspectives. 